\title{
Camouflage third-harmonic generation in the nonperturbative few-cycle regime
}

\author{
T. Tritschler ${ }^{*}, 1$, O. D. Mücke ${ }^{1}$, U. Morgner ${ }^{2}$, F. X. Kärtner ${ }^{3}$, and M. Wegener ${ }^{1}$ \\ 1 Institut für Angewandte Physik, Universität Karlsruhe (TH), Wolfgang-Gaede-Straße 1, \\ 76131 Karlsruhe, Germany \\ 2 Max-Planck-Institut für Kernphysik, Saupfercheckweg 1, 69117 Heidelberg, Germany \\ 3 Department of Electrical Engineering and Computer Science and Research Laboratory of Electronics, \\ Massachusetts Institute of Technology, Cambridge, Massachusetts 02139, USA
}

Received 24 February 2003, revised 18 March 2003, accepted 18 March 2003 Published online 25 July 2003

PACS 42.50.Md, 42.65.Re, 78.47.+p

Focusing 5 fs optical pulses, with a Rabi energy comparable to the carrier photon energy, derived from a mode-locked $\mathrm{Ti}$ : sapphire laser oscillator onto $\mathrm{ZnO}$ crystals, leads to large contributions in the emitted light, which depend on the carrier-envelope offset frequency of the laser oscillator. Moreover, we discuss that an additional contribution at twice the carrier-envelope offset frequency is due to camouflage third-harmonic generation - a process, which looks like second-harmonic generation in an inversion symmetric medium at first sight.

(C) 2003 WILEY-VCH Verlag GmbH \& Co. KGaA, Weinheim

1 Introduction With optical pulses which are short (two cycles of light) as well as intense (Rabi energy comparable to the carrier photon energy), the regime of extreme nonlinear optics in semiconductors has recently become accessible. In this regime, significant dynamics takes place on the timescale of one cycle of light and, thus, many concepts and approximations which are familiar from traditional semiconductor nonlinear optics fail [1-5]. For resonant excitation, carrier-wave Rabi flopping has recently been reported experimentally on GaAs [6-8]. In contrast to this, we review in this article our recent results on non-resonant excitation (exemplified by $\mathrm{ZnO}$ ) for which camouflage thirdharmonic generation (camouflage THG) $[9,10]$ can occur. Moreover, in this regime, the carrier-envelope offset (CEO) phase $\phi$, i.e., the phase between the rapidly oscillating carrier wave of a laser pulse and its electric field envelope, becomes an important quantity, whereas in traditional semiconductor nonlinear optics, the CEO phase is strictly irrelevant.

This article is organized as follows. In section 2 we recall a few well-known facts [11, 12] about the carrier-envelope offset frequency and its measurement in perturbative off-resonant nonlinear optics. In section 3, we discuss extreme nonlinear optics in semiconductors, i.e., the nonperturbative regime $[6-8,10]$ and compare these findings with recent experiments $[9,10]$ in section 4 before concluding in section 5 .

2 The carrier-envelope offset phase in off-resonant perturbative nonlinear optics In a modelocked laser oscillator, the CEO phase changes from pulse to pulse as group and phase velocity are generally not the same within the cavity. The corresponding frequency, the CEO frequency $f_{\phi}$, has recently started a revolution in frequency metrology $[11,12]$. In these experiments, the interference of the fundamental (with phase $\phi$ ), broadened by self-phase modulation (SPM) in a (photonic crystal)

\footnotetext{
${ }^{*}$ Corresponding author: e-mail: thorsten.tritschler@physik.uni-karlsruhe.de
} 
optical fiber, with the second-harmonic generation (SHG) with phase $2 \phi$, generated in a separate medium, leads to a beat note at frequency $f_{\phi}$ in the radio frequency (RF) spectra: Consider an incident pulse with electric field

$$
E(t)=\tilde{E}(t) \cos \left(\omega_{0} t+\phi\right) .
$$

$\tilde{E}(t)$ is the envelope of the pulse, the cosine term is the carrier wave with carrier frequency $\omega_{0}$ and CEO phase $\phi[11,12]$. In perturbative, off-resonant nonlinear optics, the optical polarization $P(t)$ of a medium can be expressed as

$$
P(t)=\epsilon_{0}\left(\chi^{(1)} E(t)+\chi^{(2)} E^{2}(t)+\chi^{(3)} E^{3}(t)+\ldots\right),
$$

with the (non)linear optical susceptibilities $\chi^{(N)}$. Consider the second-order contribution which - via the wave equation - gives rise to an electric field at carrier frequency $2 \omega_{0}$, so-called SHG, which is given by the underlined term in

$$
E_{2 \omega_{0}}(t) \propto E^{2}(t)=\tilde{E}^{2}(t) \cos ^{2}\left(\omega_{0} t+\phi\right)=\tilde{E}^{2}(t) \frac{1}{2}\left(1+\underline{\cos \left(2 \omega_{0} t+2 \phi\right)}\right) .
$$

Let us consider the resulting interference of the fundamental and the SHG contribution in frequency space. The Fourier transform of the cosine-terms have maxima at positive and at negative frequencies. We focus on the measurable positive frequency components (corresponding to the minus sign in the exponent). The resulting intensity from the interference can be written as

$$
\begin{aligned}
I_{\omega_{0}, 2 \omega_{0}}(\omega) & \propto\left|\mathrm{e}^{-\mathrm{i} \phi} \tilde{E}_{\omega_{0}}(\omega)+\mathrm{e}^{-\mathrm{i} 2 \phi} \tilde{E}_{2 \omega_{0}}(\omega)\right|^{2} \\
& =\left|\tilde{E}_{\omega_{0}}(\omega)\right|^{2}+\left|\tilde{E}_{2 \omega_{0}}(\omega)\right|^{2}+2\left|\tilde{E}_{\omega_{0}}(\omega) \tilde{E}_{2 \omega_{0}}(\omega)\right| \times \underline{\cos (\phi)} .
\end{aligned}
$$

The underlined $\cos (\phi)$-term delivers a dependence on the CEO phase $\phi$, which itself oscillates with the CEO frequency from pulse to pulse. Hence, this modulation leads to a peak at frequency $f_{\phi}$ in the spectrum. Similarly, an interference of a third-order contribution with the fundamental would lead to a peak at frequency $2 f_{\phi}$ in the spectrum.

3 The carrier-envelope offset phase in nonperturbative nonlinear optics In order to compute nonlinear optical signals from semiconductor films and their dependence on the CEO phase in a realistic manner, we have performed one-dimensional finite difference time domain calculations solving the coupled Maxwell-Bloch equations without using the rotating wave approximation, without using the slowly varying envelope approximation and accounting for actual sample structures as well as actual laser pulses and accounting for the continuum of states in the bands without using the effective mass approximation. In this article, we do not repeat these complete calculations [10] but rather focus on an intuitive understanding of the underlying processes by discussing the optical polarization $P$ obtained from the optical Bloch equations without the propagation part. Without rotating wave approximation, they are given by [13] (relaxation omitted for clarity)

$$
\left(\begin{array}{c}
\dot{u} \\
\dot{v} \\
\dot{w}
\end{array}\right)=\left(\begin{array}{ccc}
0 & +\Omega & 0 \\
-\Omega & 0 & -2 \Omega_{\mathrm{R}}(t) \\
0 & +2 \Omega_{\mathrm{R}}(t) & 0
\end{array}\right)\left(\begin{array}{c}
u \\
v \\
w
\end{array}\right),
$$

where $(u, v, w)^{\mathrm{T}}$ is the Bloch vector. Here we have introduced the optical transition frequency $\Omega$ and the Rabi frequency $\Omega_{\mathrm{R}}(t)=\hbar^{-1} \mathrm{~d} E(t)$, with the dipole matrix element $d$. Note that the Rabi frequency oscillates with the carrier frequency of light $\omega_{0}$ and, thus, periodically changes sign - quite unlike the behavior of the well-known envelope Rabi frequency $\tilde{\Omega}_{\mathrm{R}}(t)=\hbar^{-1} \mathrm{~d} \tilde{E}(t) . u(t)$ is proportional to the optical polarization $P(t), w(t)$ is the inversion.

In the numerical calculations, we take $T_{1}=\infty, T_{2}=50 \mathrm{fs}$ for the longitudinal and transverse relaxation time, respectively. These numbers are, however, not critical at all for what we want to discuss below. When relating the Rabi frequency to electric fields or intensities, we use $d=0.19 e \mathrm{~nm}$ $\left(e=1.6021 \times 10^{-19}\right.$ As is the elementary charge) obtained from $k \cdot p$ perturbation theory [13] with 

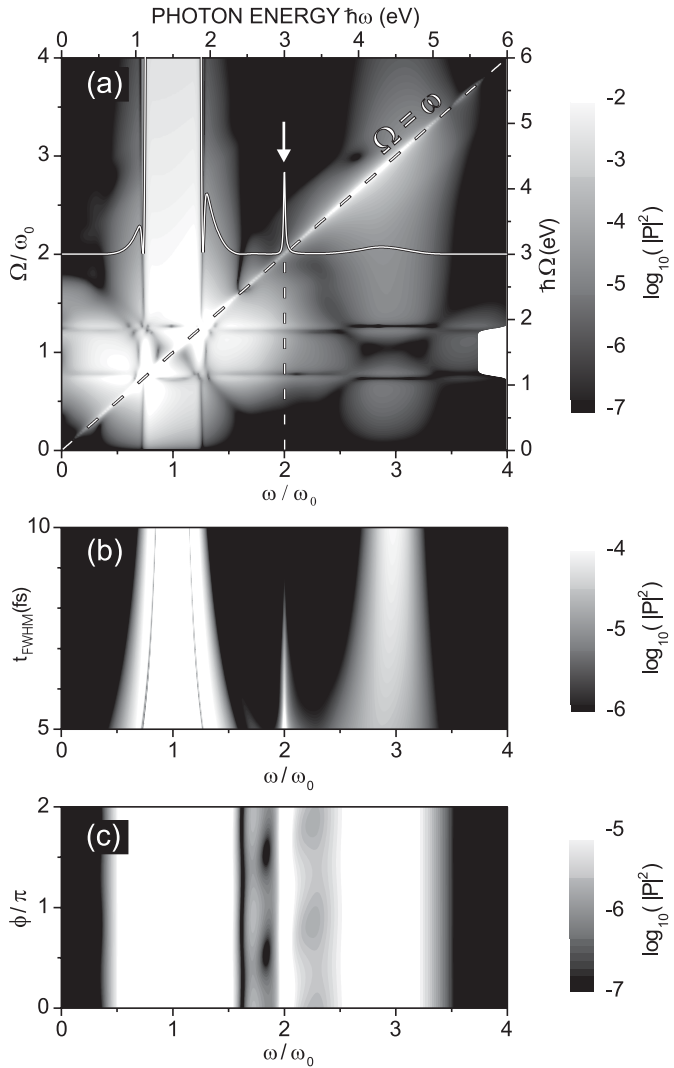

Fig. 1 Grey-scale image of the square modulus of the optical polarization $P$ (normalized) versus spectrometer frequency $\omega$ in units of the laser carrier frequency $\omega_{0}$ with $\hbar \omega_{0}=1.5 \mathrm{eV}$. The peak Rabi frequency of the exciting $\operatorname{sinc}^{2}(t)$ pulses with duration $t_{\mathrm{FWHM}}$ is $\Omega_{\mathrm{R}} / \omega_{0}=0.76$. a) $|P(\omega)|^{2}$ versus transition frequency $\Omega$ for a fixed CEO phase $\phi=0$ and $t_{\mathrm{FWHM}}=5 \mathrm{fs}$. The white curve is a cut through the data at $\Omega / \omega_{0}=2$ (linear scale). The laser pulse spectrum is shown as the white area on the RHS. b) $|P(\omega)|^{2}$ versus pulse duration $t_{\mathrm{FWHM}}$ for fixed $\Omega / \omega_{0}=2$ and $\phi=0$. c) $|P(\omega)|^{2}$ versus $\phi$ for fixed $\Omega / \omega_{0}=2$ and $t_{\text {FWHM }}=5$ fs. Taken from Ref. [10].

$\mathrm{ZnO}$ parameters. The exciting optical pulses have a duration (full width at half maximum (FWHM)) of the intensity of $t_{\mathrm{FWHM}}=5.0 \mathrm{fs}$ and are given by $E(t)=\tilde{E}(t) \cos \left(\omega_{0} t+\phi\right)$, with $\tilde{E}(t)=\tilde{E}_{0} \operatorname{sinc}\left(t / t_{0}\right)$, $t_{\mathrm{FWHM}} / t_{0}=2.7831$, and $\hbar \omega_{0}=1.5 \mathrm{eV}$, equivalent to an optical cycle with 2.8 fs period ${ }^{1}$. The corresponding intensity spectrum is depicted by the white area on the RHS of Fig. 1a. Figure 1a shows the square modulus of the optical polarization $P$ as a function of spectrometer frequency $\omega$ and transition frequency $\Omega$. Both cover the range from 0 to $6 \mathrm{eV}$ (the $\mathrm{ZnO}$ room temperature band gap is at $3.3 \mathrm{eV}$ ), the logarithmic grey-scale ranges down to seven orders of magnitude below the peak. In Fig. 1, the peak envelope Rabi energy $\hbar \Omega_{\mathrm{R}}$ is given by $\Omega_{\mathrm{R}} / \omega_{0}=0.76$ which is equivalent to a peak of the electric field envelope of $\tilde{E}_{0}=6 \times 10^{9} \mathrm{~V} / \mathrm{m}$ or a peak intensity in vacuum of $I=5 \times 10^{12} \mathrm{~W} / \mathrm{cm}^{2}$ (see corresponding discussion in section 4).

The signal contribution around the $\Omega=\omega$ line in Fig. 1a exhibits a constriction, the exact position of which depends on the Rabi frequency. Let us start the discussion with the part above this constriction and consider a transition frequency $\Omega$ on the vertical axis at twice the laser carrier frequency $\omega_{0}$, i.e., at $\Omega / \omega_{0}=2$ in Fig. 1a. Here we observe a well-defined peak in the optical spectra right at spectrometer frequency $\omega=2 \omega_{0}$ (see white curve in Fig. 1a which is a cut through the data at $\Omega / \omega_{0}=2$ plotted on a linear scale). What is the origin of this peak? It cannot be second-harmonic generation in the strict sense, because that is forbidden by symmetry. A part of it is the resonant enhancement way down in the low-energy tail of the third harmonic of the laser photon energy. We want to call this contribution camouflage third-harmonic generation. It looks like SHG but actually is not SHG - "a wolf in a sheep's clothing”. For pulses containing many cycles of light, this contribution is expected to disappear because of negligible overlap of the third-harmonic response function and the resonance - it is specific for the regime of few-cycle pulses. This is illustrated by the dependence on pulse duration depicted in Fig. $1 \mathrm{~b}$. Here, the pulse duration $t_{\mathrm{FWHM}}$ covers the range from $5 \mathrm{fs}$ to $10 \mathrm{fs}$, very roughly equivalent to two-cycle pulses to four-cycle pulses. The camouflage THG almost vanishes for a three-cycle long incident optical pulse.

\footnotetext{
${ }^{1}$ The long-time tails have been suppressed by a Gaussian, i.e., $E(t)=\tilde{E}_{0} \operatorname{sinc}\left(t / t_{0}\right) \exp \left(-t^{2} / t_{\text {Gauss }}^{2}\right) \times \cos \left(\omega_{0} t+\phi\right)$ with $t_{\text {Gauss }}=28 \mathrm{fs}$.
} 
Figure 1c shows the dependence on the CEO phase $\phi$ for a selected transition frequency of $\Omega / \omega_{0}=2$. All other parameters are as in Fig. 1a. It becomes obvious that a part of the interference occurs in between the fundamental, i.e. $\omega / \omega_{0}=1$, and $\omega / \omega_{0}=2$. Note that the period of the signal versus $\phi$ is $\pi$ rather than $2 \pi$, equivalent to a peak at frequency $2 f_{\phi}$ in the RF spectrum, originating from the interference of the fundamental (with phase $\phi$ ) and the third harmonic (with phase $3 \phi$ ), with corresponding difference phase $2 \phi$. The usual SHG would appear in the same region in the optical spectra as the camouflage THG, but its phase is $2 \phi$ rather than $3 \phi$, thus, it leads to a peak at frequency $f_{\phi}$ in the RF spectra when beating with the fundamental. Another part of the interference in Fig. 1c occurs in between $\omega / \omega_{0}=2$ and $\omega / \omega_{0}=3$. This shows that the camouflage peak around $\omega / \omega_{0}=2$ in Fig. 1a is indeed a mixture of resonantly enhanced "SPM" and resonantly enhanced THG.

Camouflage THG becomes significant only for short and intense optical pulses. The latter aspect becomes evident from the dependence on peak Rabi energy $\Omega_{\mathrm{R}}$ depicted in Fig. 2 . Amazingly, for very large peak Rabi energies approaching $\Omega_{\mathrm{R}} / \omega_{0}=1.3$, the camouflage THG peak at $\omega / \omega_{0}=2$ becomes even larger than the usual THG at $\omega / \omega_{0}=3$.

This resonant enhancement we have just described is clearly different from the behavior around $\Omega / \omega_{0} \approx 1$ on the vertical axis of Fig. 1a, which is due to carrier-wave Rabi flopping, i.e., due to the large splitting of the sidebands centered around $\omega / \omega_{0}=1$ and $\omega / \omega_{0}=3$, arising from a modulation of the two-level system with the Rabi frequency as described in detail in Refs. [6-8]. There, theory also predicts a dependence on the CEO phase with period $\pi$ rather than $2 \pi$ as a result of the interference of different Mollow sidebands [7]. Corresponding experiments [8] have also been performed.

Finally, note that the off-resonant limit which we have discussed in section 2 is hardly recovered. This is especially true for large laser intensities, i.e., for large values of $\Omega_{\mathrm{R}} / \omega_{0}$. Reaching the offresonant limit would mean that the shape of the spectrum (not necessarily the strength) becomes independent on the transition energy $\hbar \Omega$. In Fig. 1a, this limit is not yet reached even at $\hbar \Omega=6 \mathrm{eV}$. Remember that there are very few materials, the lowest transitions of which are beyond that number. In other words: The limit of off-resonant excitation becomes highly questionable at large laser intensities.

4 Experiments with 5 fs pulses on ZnO In our experiments [9], 5 fs linearly polarized optical pulses [14] from a mode-locked laser oscillator operating at $f_{\mathrm{r}}=81 \mathrm{MHz}$ repetition frequency are tightly focused onto a $\mathrm{ZnO}$ crystal by means of a high numerical aperture $(\mathrm{NA}=0.5)$ reflective microscope objective. This leads to a spot radius of about one micrometer.

Let us briefly convince ourselves that average powers of some ten $\mathrm{mW}$ lead to very large intensities or Rabi energies. Assume we have an average power of $64 \mathrm{~mW}$ in front of the sample. At the above repetition frequency this corresponds to a pulse energy of $0.8 \mathrm{~nJ}$ and to a pulse power of $0.8 \mathrm{~nJ} / 5 \mathrm{fs}=158 \mathrm{~kW}$. With a spot area of $\pi\left(10^{-4} \mathrm{~cm}\right)^{2}$, this leads to an intensity of

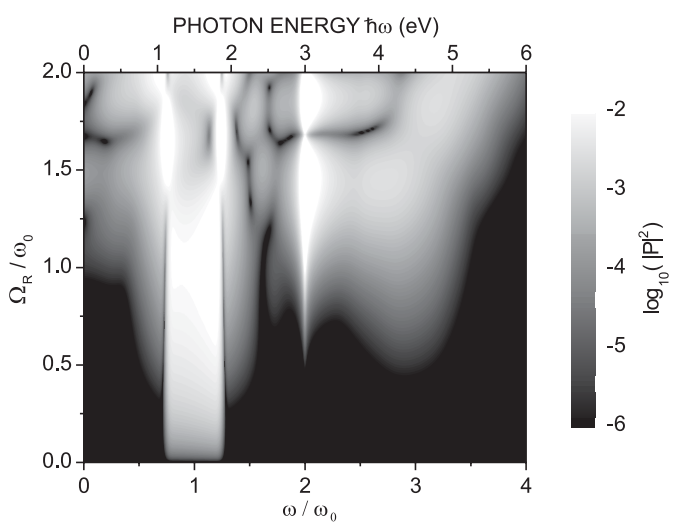

Fig. 2 Grey-scale image of the square modulus of the optical polarization $P$ (normalized) versus peak Rabi frequency $\Omega_{\mathrm{R}}$ in units of the laser carrier frequency $\omega_{0}$ for fixed $\Omega / \omega_{0}=2, \phi=0$ and $t_{\mathrm{FWHM}}=5 \mathrm{fs}$, see Fig. 1 . 
$I=5 \times 10^{12} \mathrm{~W} / \mathrm{cm}^{2}$. With

$$
I=\frac{1}{2} \sqrt{\frac{\varepsilon_{0}}{\mu_{0}}} \tilde{E}_{0}^{2}
$$

$\left(\mu_{0}=4 \pi \times 10^{-7} \mathrm{VsA}^{-1} \mathrm{~m}^{-1}\right.$ and $\left.\varepsilon_{0}=8.8542 \times 10^{-12} \mathrm{AsV}^{-1} \mathrm{~m}^{-1}\right)$ this translates into a peak of the electric field envelope of $\tilde{E}_{0}=6 \times 10^{9} \mathrm{~V} / \mathrm{m}$ and with the dipole matrix element $d=0.19$ e nm into a peak of the (envelope) Rabi energy $\hbar \Omega_{\mathrm{R}}=\mathrm{d} \tilde{E}_{0}=1.14 \mathrm{eV}$. With $\hbar \omega_{0}=1.5 \mathrm{eV}$ we get $\Omega_{\mathrm{R}} / \omega_{0}=0.76$. This is exactly the number we have used in Fig. 1.

Remember that $\mathrm{ZnO}$ is birefringent and that it has no inversion symmetry. The room temperature $\mathrm{ZnO}$ band gap energy is $E_{\mathrm{g}}=3.3 \mathrm{eV}$. Amazingly, the precise value of the band gap energy of $\mathrm{ZnO}$ was subject of scientific discussions until rather recently [15]. Figure 3a shows the measured RF power spectrum for a $100 \mu \mathrm{m}$ thick single crystal with the crystallographic $c$-axis in the plane of the platelet and for polarization $\boldsymbol{E} \| \boldsymbol{c}$, Fig. $3 \mathrm{~b}$ that of a $350 \mathrm{~nm}$ thin epitaxial film of $\mathrm{ZnO}$ where the $\boldsymbol{c}$-axis is perpendicular to the film with polarization $\boldsymbol{E} \perp \boldsymbol{c}$. For the thick sample, we observe a rather large peak at the CEO frequency $f_{\phi}$. At an average power of merely $64 \mathrm{~mW}$ and at $10 \mathrm{kHz}$ bandwidth, it is more than $30 \mathrm{~dB}$ above the noise floor. On another sample we have observed more than $35 \mathrm{~dB}$ under similar conditions. This simple arrangement - in which fs-pulses are focused onto the crystal and the transmitted light is detected - might prove useful for measuring and/or stabilizing the CEO frequency of mode-locked laser oscillators. (In particular, one avoids the use of a (microstructured) optical fiber and of an interferometer, both of which are major sources of noise, as is discussed in the corresponding recent literature.) This is true also for longer pulses, see Ref. [9]. Moreover, the data of Fig. 3a also show weak contributions at $2 f_{\phi}$ and $\left(f_{r}-2 f_{\phi}\right)$. Following our discussion in section 2 , they are due to an interference of the fundamental broadened by "SPM" and the third harmonic. These contributions become even more prominent for the $350 \mathrm{~nm}$ thin sample, see Fig. $3 \mathrm{~b}$. Here, the $2 f_{\phi}$ peak is even larger than the $f_{\phi}$ peak. Specifically, as described in detail in section 3 , the $2 f_{\phi}$ peak originates from a resonant enhancement of the third harmonic at the $\mathrm{ZnO}$ band gap, i.e., it is due to camouflage third-harmonic generation, while the $f_{\phi}$ peak is the conventional beating of the "SPM" contribution with the SHG. A much more detailed and direct comparison between experiment and theory is given in Ref. [10]. This reference also comprises interferometric optical spectra, which help to distinguish different models by giving insight into the dynamics of the contributions.

What do we learn from this discussion in terms of the problem of determining the CEO phase itself from such a measurement? It is quite obvious that the $2 f_{\phi}$ contribution is rather disturbing at this

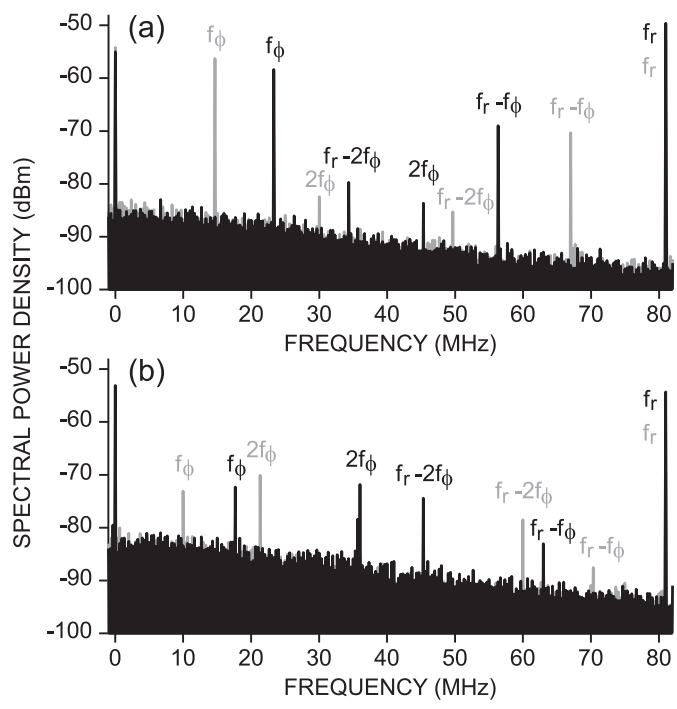

Fig. 3 RF power spectra, $10 \mathrm{kHz}$ resolution and video bandwidth, $I=5 \times 10^{12} \mathrm{~W} / \mathrm{cm}^{2}$. a) $100 \mu \mathrm{m}$ thick $\mathrm{ZnO}$ single crystal, $455-480 \mathrm{~nm}$ optical filter, b) $350 \mathrm{~nm}$ thin $\mathrm{ZnO}$ epitaxial layer, $455-500 \mathrm{~nm}$ optical filter. The peaks at the repetition frequency $f_{r}$, the carrier-envelope offset frequency $f_{\phi}$, its second harmonic $2 f_{\phi}$ and the mixing products $\left(f_{r}-f_{\phi}\right)$ and $\left(f_{r}-2 f_{\phi}\right)$ are labeled. The black and grey data correspond to slightly different swivel mirror (high reflector in the prism arm of the laser cavity) positions, respectively. When removing intracavity prism material $\left(\mathrm{CaF}_{2}\right)$, the $f_{\phi}$ and $2 f_{\phi}\left(\left(f_{r}-f_{\phi}\right)\right.$ and $\left.\left(f_{r}-2 f_{\phi}\right)\right)$ peaks shift to the left (right). Taken from Ref. [9]. 
point. While its physics is quite interesting, the beat signal is at best (i.e., neglecting phase shifts) a superposition of a $\cos (\phi)$-oscillation and a $\cos (2 \phi)$-oscillation. As the pre-factors are expected to depend on excitation intensity, it becomes rather unpleasant to determine $\phi$ from the beat signal although it is not impossible. Section 3 has shown two possibilities to get rid of the $2 f_{\phi}$ contribution: Reduce the excitation intensity and/or restrict yourself to longer pulses. The first option obviously also reduces the signal strength, the latter not too much - although short pulses are the most interesting to be characterized. Either option is still in reach. Remember that the RF power spectrum in Fig. 3 has been measured with a mode-locked laser oscillator, the CEO frequency of which was not stabilized. Stabilizing the CEO frequency along the lines of Refs. [11, 12] would allow for much longer integration times, thus allow for smaller excitation intensities. Furthermore, somewhat thicker samples might still be acceptable in terms of their distortion of the CEO phase of the incident pulses within the sample. Let us have a closer look at this aspect. Generally, we have for the linear optical shift, $\delta \phi$, of the CEO phase in a sample of thickness $l$ at a center wavelength $\lambda_{0}=2 \pi c_{0} / \omega_{0} \quad\left(c_{0}\right.$ is the vacuum velocity of light)

$$
\delta \phi=2 \pi \frac{\left(\frac{l}{v_{\text {group }}}-\frac{l}{v_{\text {phase }}}\right)}{2 \pi / \omega_{0}}=-2 \pi \frac{\mathrm{d} n}{\mathrm{~d} \lambda}\left(\lambda_{0}\right) l,
$$

with the vacuum-wavelength $\lambda$ dependent refractive index $n(\lambda)$. The latter can be expressed via the Sellmeier coefficients. From the known Sellmeier coefficients of $\mathrm{ZnO}$ [16], we derive after some straightforward but tedious mathematics

$$
\delta \phi_{\mathrm{ZnO}, \lambda_{0}=826 \mathrm{~nm}}=0.013 \times 2 \pi l / 100 \mathrm{~nm} .
$$

For the $l=350 \mathrm{~nm}$ thin $\mathrm{ZnO}$ epitaxial film, $\delta \phi$ is merely $4.6 \%$ of $2 \pi$. Furthermore, the nonlinear optical shift due to self-phase modulation is estimated [9] to be even smaller than this, with $1 \%$ of $2 \pi$ at an intensity of $I=5.0 \times 10^{12} \mathrm{~W} / \mathrm{cm}^{2}$.

The question which remains to be answered at the end of the day is: How sensitive is the phase of the beat signal on the precise choice of the optical detection frequency, on excitation intensity, on details of the sample design, antireflection coatings [7], approximations of the model, etc. ? At this point, more advanced microscopic theory accounting for correlations and scattering on a quantum kinetic footing would be really important. As the (coherent) carrier densities are very large, Coulomb effects are expected to be more important than phonon effects. Alternatively, one could calibrate, e.g., the $\mathrm{ZnO}$ beat signal with a reference, i.e., with another independent and established method to determine the CEO phase itself. Such a method does, however, presently not exist.

5 Conclusions Exciting $\mathrm{ZnO}$ with intense $5 \mathrm{fs}$ optical pulses, we find a large peak at the carrierenvelope offset frequency ( $>35 \mathrm{~dB}$ at $10 \mathrm{kHz}$ bandwidth and at $64 \mathrm{~mW}$ average power) in the radio frequency power spectra of light emitted into the forward direction. In addition, we observe a peak at twice the carrier-envelope offset frequency, which is attributed to camouflage third-harmonic generation - third-harmonic generation, which looks like second-harmonic generation at first sight. This process can lead to frequency doubling in an inversion-symmetric medium.

Acknowledgements The work of M. W. is supported by projects DFG-We 1497/11-1 and DFG-We 1497/9-1, that of U. M. by project DFG-Mo 850/2-1 and that of F. X. K. by ONR and NSF grant ECS-0217358.

\section{References}

[1] R. Bavli and H. Metiu, Phys. Rev. Lett. 69, 1986 (1992).

[2] M. Yu. Ivanov, P. B. Corkum, and P. Dietrich, Laser Phys. 3, 375 (1993).

[3] A. Levinson, M. Segev, G. Almogy, and A. Yariv, Phys. Rev. A 49, R 661 (1994).

[4] T. Zuo, S. Chelkowski, and A. D. Bandrauk, Phys. Rev. A 49, 3943 (1994). 
[5] S. Hughes, Phys. Rev. Lett. 81, 3363 (1998).

[6] O. D. Mücke, T. Tritschler, M. Wegener, U. Morgner, and F. X. Kärtner, Phys. Rev. Lett. 87, 057401 (2001).

[7] O. D. Mücke, T. Tritschler, M. Wegener, U. Morgner, and F. X. Kärtner, Phys. Rev. Lett. 89, 127401 (2002).

[8] O. D. Mücke, T. Tritschler, M. Wegener, U. Morgner, F. X. Kärtner, G. Khitrova, and H. M. Gibbs, unpublished (2003).

[9] O. D. Mücke, T. Tritschler, M. Wegener, U. Morgner, and F. X. Kärtner, Opt. Lett. 27, 2127 (2002).

[10] T. Tritschler, O. D. Mücke, M. Wegener, U. Morgner, and F. X. Kärtner, Phys. Rev. Lett., 90, 217404 (2003).

[11] A. Apolonski, A. Poppe, G. Tempea, Ch. Spielmann, Th. Udem, R. Holzwarth, T. W. Hänsch, and F. Krausz, Phys. Rev. Lett. 85, 740 (2000).

[12] D. J. Jones, S. A. Diddams, J. K. Ranka, A. Stentz, R. S. Windeler, J. L. Hall, and S. T. Cundiff, Science 288, 635 (2000).

[13] W. Schäfer and M. Wegener, Semiconductor Optics and Transport Phenomena, Advanced Texts in Physics (Springer, Berlin and Heidelberg, 2002).

[14] U. Morgner, F. X. Kärtner, S. H. Cho, Y. Chen, H. A. Haus, J. G. Fujimoto, E. P. Ippen, V. Scheuer, G. Angelow, and T. Tschudi, Opt. Lett. 24, 411 (1999).

[15] V. Srikant and D. R. Clarke, J. Appl. Phys. 83, 5447 (1998).

[16] X. W. Sun and H. S. Kwok, J. Appl. Phys. 86, 408 (1999). 